\title{
Kinetic Theory of Simple Reacting Spheres I
}

\author{
Jacek Polewczak ${ }^{\mathrm{a}}$, Ana Jacinta Soares ${ }^{\mathrm{b}}$ \\ a Department of Mathematics, California State University, Northridge, California 91330, USA \\ b Departamento de Matemática e Aplicações, Universidade do Minho, Braga, Portugal
}

\begin{abstract}
We consider physical and mathematical aspects of the model of simple reacting spheres (SRS) in the kinetic theory of chemically reacting fluids. The SRS, being a natural extension of the hard-sphere collisional model, reduces itself to the revised Enskog theory when the chemical reactions are turned off. In the dilute-gas limit, it provides an interesting kinetic model of chemical reactions that has not been considered before. In contrast to other reactive kinetic theories (e.g., line-of-centers models), the SRS has built-in detailed balance and microscopic reversibility conditions. The mathematical analysis of the work consists of global existence result for the system of partial differential equations for the model of SRS.
\end{abstract}

\section{Simple Reacting Spheres}

Simple reacting spheres (SRS) has been developed by N. Xystris, J. S. Dahler [1] and further advanced by J. S. Dahler and L. Quin in [2], [3]. The present paper is the first in a series of our articles on physical and mathematical properties of SRS. In the SRS model, the molecules behave as if they were single mass points with two internal states of excitation. Collisions may alter the internal states: this occurs when the kinetic energy associated with the reactive motion exceeds the activation energy. Reactive and non-reactive collision events are considered to be hard spheres-like. In a four component mixture $A, B, A^{*}, B^{*}$, the chemical reactions are of the type: $A+B \rightleftharpoons A^{*}+B^{*}$. Here, $A^{*}$ and $B^{*}$ are distinct species from $A$ and $B$. We use the indices $1,2,3$, and 4 for the particles $A, B, A^{*}$, and $B^{*}$ respectively. Furthermore, $m_{i}$ and $d_{i}$ denote the mass and the diameter of the i-th particle, $i=1, \ldots, 4$, and reactions take place when the reactive particles are separated by a distance $\sigma_{12}=\frac{1}{2}\left(d_{1}+d_{2}\right)$ or $\sigma_{34}=\frac{1}{2}\left(d_{3}+d_{4}\right)$. The conservation of mass has the form $m_{1}+m_{2}=m_{3}+m_{4}=M$. Reactions take place when the reactive particles are separated by a distance $\sigma_{12}=\frac{1}{2}\left(d_{1}+d_{2}\right)$, where $d_{i}$ denotes the diameter of the i-th particle.

\section{$1.1 \quad$ Elastic encounters}

In the case of elastic collisions between a pair of particles from species $i$ and $s$, the initial velocities $v, w$ take post-collisional values

$$
v^{\prime}=v-2 \frac{\mu_{i s}}{m_{i}} \epsilon\langle\epsilon, v-w\rangle, \quad w^{\prime}=w+2 \frac{\mu_{i s}}{m_{s}} \epsilon\langle\epsilon, v-w\rangle .
$$

Here, $\langle\cdot, \cdot\rangle$ is the inner product in $\mathbb{R}^{3}, \epsilon$ is a vector along the line passing through the centers of the spheres at the moment of impact, i.e., $\epsilon \in \mathbb{S}_{+}^{2}=\left\{\epsilon \in \mathbb{R}^{3}:|\epsilon|=1,\langle\epsilon, v-w\rangle \geq 0\right\}$ 
and $\mu_{i s}=m_{i} m_{s} /\left(m_{i}+m_{s}\right)$ is the reduced mass of the colliding pair. $m_{i}$ and $m_{s}$ are the masses of particles from i-th and s-th species, respectively $(i, s=1,2,3,4)$.

\section{$1.2 \quad$ Reactive encounters}

For the reactive collision between particles from species $i$ and $s$ to occur $(i, s=1, \ldots, 4)$, the kinetic energy associated with the relative motion along the line of centers must exceed the activation energy $\gamma_{i}$,

$$
(1 / 2) \mu_{i s}(\langle\epsilon, v-w\rangle)^{2} \geq \gamma_{i}
$$

In the case of the (endothermic) reaction $A+B \rightarrow A^{*}+B^{*}$ the velocities $v, w$ take their post-reactive values

$$
\begin{aligned}
& v^{\ddagger}=\frac{1}{M}\left[m_{1} v+m_{2} w+m_{4} \sqrt{\frac{\mu_{12}}{\mu_{34}}}\left\{(v-w)-\epsilon\langle\epsilon, v-w\rangle+\epsilon \alpha^{-}\right\}\right], \\
& w^{\ddagger}=\frac{1}{M}\left[m_{1} v+m_{2} w-m_{3} \sqrt{\frac{\mu_{12}}{\mu_{34}}}\left\{(v-w)-\epsilon\langle\epsilon, v-w\rangle+\epsilon \alpha^{-}\right\}\right],
\end{aligned}
$$

with $\alpha^{-}=\sqrt{(\langle\epsilon, v-w\rangle)^{2}-2 E_{a b s} / \mu_{12}}$, and $E_{a b s}$ the energy absorbed by the internal degrees of freedom. The absorbed energy $E_{a b s}$ has the property $E_{a b s}=E_{3}+E_{4}-E_{1}-E_{2}>$ 0 , where $E_{i}>0, i=1, \ldots 4$, is the energy of i-th particle associated with its internal degrees of freedom.

Now, in order to complete the definition of the model, the activation energies $\gamma_{1}, \gamma_{2}$ for $A$ and $B$ are chosen to satisfy $\gamma_{1} \geq E_{a b s}>0$, and by symmetry, $\gamma_{2}=\gamma_{1}$.

For the inverse (exothermic) reaction, $A^{*}+B^{*} \rightarrow A+B$, the post-reactive velocities are given by

$$
\begin{aligned}
& v^{\dagger}=\frac{1}{M}\left[m_{3} v+m_{4} w+m_{2} \sqrt{\frac{\mu_{34}}{\mu_{12}}}\left\{(v-w)-\epsilon\langle\epsilon, v-w\rangle+\epsilon \alpha^{+}\right\}\right], \\
& w^{\dagger}=\frac{1}{M}\left[m_{3} v+m_{4} w-m_{1} \sqrt{\frac{\mu_{34}}{\mu_{12}}}\left\{(v-w)-\epsilon\langle\epsilon, v-w\rangle+\epsilon \alpha^{+}\right\}\right],
\end{aligned}
$$

with $\alpha^{+}=\sqrt{(\langle\epsilon, v-w\rangle)^{2}+2 E_{a b s} / \mu_{34}}$, and the activation energies for $A^{*}$ and $B^{*}$ being $\gamma_{3}=\gamma_{1}-E_{a b s}$ and $\gamma_{4}=\gamma_{3}$.

Post- and pre-collisional velocities of the reactive pairs satisfy conservation of the momentum

$$
m_{1} v+m_{2} w=m_{3} v^{\ddagger}+m_{4} w^{\ddagger}, \quad m_{3} v+m_{4} w=m_{1} v^{\dagger}+m_{2} w^{\dagger} .
$$

A part of kinetic energy is exchanged with the energy absorbed by the internal states. The following equalities hold:

$$
m_{1} v^{2}+m_{2} w^{2}=m_{3} v^{\ddagger 2}+m_{4} w^{\ddagger 2}+2 E_{a b s}, \quad m_{3} v^{2}+m_{4} w^{2}=m_{1} v^{\dagger 2}+m_{2} w^{\dagger 2}-2 E_{a b s} .
$$

\subsection{The system of equations}

For $i=1,2,3,4, f_{i}(t, x, v)$ denotes the one-particle distribution function of the ith component of the reactive mixture. The function $f_{i}(t, x, v)$, which changes in time due to free 
streaming and collisions (elastic and reactive), represents at time $t$ the number density of particles at point $x$ with velocity $v$.

The SRS kinetic system has the form

$$
\frac{\partial f_{i}}{\partial t}+v \frac{\partial f_{i}}{\partial x}=J_{i}^{E}+J_{i}^{R}, \quad i=1,2,3,4,
$$

where $J_{i}^{E}$ is the non-reactive (hard-sphere) collision operator

$$
\begin{gathered}
J_{i}^{E}=\sum_{s=1}^{4}\left\{\sigma_{i s}^{2} \iint_{\mathbb{R}^{3} \times \mathbb{S}_{+}^{2}}\left[f_{i s}^{(2)}\left(t, x, v^{\prime}, x-\sigma_{i s} \epsilon, w^{\prime}\right)-f_{i s}^{(2)}\left(t, x, v, x+\sigma_{i s} \epsilon, w\right)\right]\langle\epsilon, v-w\rangle d \epsilon d w\right\} \\
-\beta_{i j} \sigma_{i j}^{2} \iint_{\mathbb{R}^{3} \times \mathbb{S}_{+}^{2}}\left[f_{i j}^{(2)}\left(t, x, v^{\prime}, x-\sigma_{i j} \epsilon, w^{\prime}\right)-f_{i j}^{(2)}\left(t, x, v, x+\sigma_{i j} \epsilon, w\right)\right] \\
\times \Theta\left(\langle\epsilon, v-w\rangle-\Gamma_{i j}\right)\langle\epsilon, v-w\rangle d \epsilon d w
\end{gathered}
$$

and $f_{i s}^{(2)}$ approximates the density of pairs of particles in collisional configurations. The second term in (10), with $\beta_{i j}$ in front of it, singles out those pre-collisional states that are energetic enough to result in the reaction, and thus preventing double counting of the events in the collisional integrals. In the case when $\beta_{i j}=0$, for $i, j=1, \ldots, 4$, the term $J_{i}^{E}$, in (10), reduces to two-particle collisional operator for 4-species mixtures with hard-sphere potential. For $i=1,2,3,4$, the reactive terms are

$$
\begin{gathered}
J_{i}^{R}=\beta_{i j} \sigma_{i j}^{2} \iint_{\mathbb{R}^{3} \times \mathbb{S}_{+}^{2}}\left[\left(\frac{\mu_{i j}}{\mu_{k l}}\right)^{3 / 2} f_{k l}^{(2)}\left(t, x, v_{i j}^{\odot}, x-\sigma_{i j} \epsilon, w_{i j}^{\odot}\right)-f_{i j}^{(2)}\left(t, x, v, x+\sigma_{i j} \epsilon, w\right)\right] \\
\times \Theta\left(\langle\epsilon, v-w\rangle-\Gamma_{i j}\right)\langle\epsilon, v-w\rangle d \epsilon d w .
\end{gathered}
$$

Here, $0 \leq \beta_{i j} \leq 1$ are the steric factors, $\Gamma_{i j}=\sqrt{2 \gamma_{i} / \mu_{i j}}$, and $\Theta$ is the Heaviside step function. The pairs of post-reactive velocities are $\left(v_{i j}^{\odot}, w_{i j}^{\odot}\right)=\left(v^{\ddagger}, w^{\ddagger}\right)$ for $i, j=1,2$, and $\left(v_{i j}^{\odot}, w_{i j}^{\odot}\right)=\left(v^{\dagger}, w^{\dagger}\right)$ for $i, j=3,4$. Pairs of indices $(i, j)$ and $(k, l)$ are from the set of quadruples $(i, j, k, l):\{(1,2,3,4),(2,1,4,3),(3,4,1,2),(4,3,2,1)\}$.

\section{Lemma 1.}

(1) For $i, s=1,2,3,4$, the inverse velocities to $v^{\prime}, w^{\prime}$ are given by

$$
v=v^{\prime}-2 \frac{\mu_{i s}}{m_{i}} \epsilon\left\langle\epsilon, v^{\prime}-w^{\prime}\right\rangle, \quad w=w^{\prime}+2 \frac{\mu_{i s}}{m_{s}} \epsilon\left\langle\epsilon, v^{\prime}-w^{\prime}\right\rangle .
$$

For fixed $\epsilon$, the Jacobian of the transformation $(v, w) \mapsto\left(v^{\prime}, w^{\prime}\right)$ is equal to -1 . Furthermore, $\left\langle\epsilon, v^{\prime}-w^{\prime}\right\rangle=-\langle\epsilon, v-w\rangle$,

(2) The inverse velocities to $v^{\ddagger}, w^{\ddagger}$ are given by

$$
\begin{aligned}
& v=\frac{1}{M}\left[m_{3} v^{\ddagger}+m_{4} w^{\ddagger}+m_{2} \sqrt{\frac{\mu_{34}}{\mu_{12}}}\left\{\left(v^{\ddagger}-w^{\ddagger}\right)-\epsilon\left\langle\epsilon, v^{\ddagger}-w^{\ddagger}\right\rangle+\epsilon \alpha^{+}\right\}\right], \\
& w=\frac{1}{M}\left[m_{3} v^{\ddagger}+m_{4} w^{\ddagger}-m_{1} \sqrt{\frac{\mu_{34}}{\mu_{12}}}\left\{\left(v^{\ddagger}-w^{\ddagger}\right)-\epsilon\left\langle\epsilon, v^{\ddagger}-w^{\ddagger}\right\rangle+\epsilon \alpha^{+}\right\}\right],
\end{aligned}
$$


and the inverse velocities to $v^{\dagger}, w^{\dagger}$ are given by

$$
\begin{aligned}
& v=\frac{1}{M}\left[m_{1} v^{\dagger}+m_{2} w^{\dagger}+m_{4} \sqrt{\frac{\mu_{12}}{\mu_{34}}}\left\{\left(v^{\dagger}-w^{\dagger}\right)-\epsilon\left\langle\epsilon, v^{\dagger}-w^{\dagger}\right\rangle+\epsilon \alpha^{-}\right\}\right], \\
& w=\frac{1}{M}\left[m_{1} v^{\dagger}+m_{2} w^{\dagger}-m_{3} \sqrt{\frac{\mu_{12}}{\mu_{34}}}\left\{\left(v^{\dagger}-w^{\dagger}\right)-\epsilon\left\langle\epsilon, v^{\dagger}-w^{\dagger}\right\rangle+\epsilon \alpha^{-}\right\}\right] .
\end{aligned}
$$

(3) For fixed $\epsilon$, the Jacobians of the transformations $(v, w) \mapsto\left(v^{\dagger}, w^{\dagger}\right)$ and $(v, w) \mapsto\left(v^{\ddagger}, w^{\ddagger}\right)$ are given by

$$
\left(\frac{\mu_{34}}{\mu_{12}}\right)^{3 / 2} \frac{\langle\epsilon, v-w\rangle}{\alpha^{+}} \text {and }\left(\frac{\mu_{12}}{\mu_{34}}\right)^{3 / 2} \frac{\langle\epsilon, v-w\rangle}{\alpha^{-}} \text {, }
$$

respectively.

(4) Furthermore, $\left\langle\epsilon, v^{\dagger}-w^{\dagger}\right\rangle=\alpha^{+},\left\langle\epsilon, v^{\ddagger}-w^{\ddagger}\right\rangle=\alpha^{-}, \frac{1}{2} \mu_{12}(\langle\epsilon, v-w\rangle)^{2}-\gamma_{1}=$ $\frac{1}{2} \mu_{34}\left(\left\langle\epsilon, v^{\ddagger}-w^{\ddagger}\right\rangle\right)^{2}-\gamma_{3}$, and $\frac{1}{2} \mu_{34}(\langle\epsilon, v-w\rangle)^{2}-\gamma_{3}=\frac{1}{2} \mu_{12}\left(\left\langle\epsilon, v^{\dagger}-w^{\dagger}\right\rangle\right)^{2}-\gamma_{1}$.

Due to space limitations, the proof of Lemma 1 will be provided in the forthcoming work.

\section{The dilute SRS kinetic system}

The system of equations (9)-(11) requires a closure relation for $f_{i s}^{(2)}$. In the case of moderately dense gases, the two-particle distribution function $f_{i s}^{(2)}$ is usually approximated by

$$
f_{i s}^{(2)}\left(t, x_{1}, v_{1}, x_{2}, v_{2}\right)=g_{i s}^{(2)}\left(x_{1}, x_{2} \mid\left\{n_{i}(t, \cdot)\right\}\right) f_{i}\left(t, x_{1}, v_{1}\right) f_{s}\left(t, x_{2}, v_{2}\right),
$$

where $n_{i}(t, x)=\int_{\mathbb{R}^{3}} f_{i}(t, x, v) d v$ is the local number density of the component $i$ and $g_{i j}^{(2)}$ is the known pair correlation function for a non-uniform hard-sphere system at equilibrium with the local densities $n_{i}(t, x)$. The notation $g_{i j}^{(2)}\left(x_{1}, x_{2} \mid\left\{n_{i}(t, \cdot)\right\}\right)$ indicates that $g_{i j}^{(2)}$ is a functional of the local densities $n_{i}$. The closure relation (18) is employed in [2] and [3]. Finally, in the case of non-reactive mixtures $\left(\beta_{i j}=0\right.$, for $\left.i, j=1, \ldots 4\right)$, the corresponding system of equations (9)-(11) becomes the revised Enskog system for 4-species mixtures [4]. In this work, we will consider a dilute gas regime with the corresponding closure relation given by:

$$
f_{i s}^{(2)}\left(t, x_{1}, v_{1}, x_{2}, v_{2}\right)=f_{i}\left(t, x_{1}, v_{1}\right) f_{s}\left(t, x_{2}, v_{2}\right) .
$$

The system of equations (9)-(11) takes the form:

$$
\frac{\partial f_{i}}{\partial t}+v \frac{\partial f_{i}}{\partial x}=J_{i}^{E}+J_{i}^{R}, \quad f_{i}(0, x, v)=f_{i 0}(x, v), \quad i=1, \ldots, 4, \quad(x, v) \in \Omega \times \mathbb{R}^{3},
$$

with

$$
\begin{aligned}
J_{i}^{E} & =\sum_{s=1}^{4}\left\{\sigma_{i s}^{2} \iint_{\mathbb{R}^{3} \times \mathbb{S}_{+}^{2}}\left[f_{i}\left(t, x, v^{\prime}\right) f_{s}\left(t, x, w^{\prime}\right)-f_{i}(t, x, v) f_{s}(t, x, w)\right]\langle\epsilon, v-w\rangle d \epsilon d w\right\} \\
& -\beta_{i j} \sigma_{i j}^{2} \iint_{\mathbb{R}^{3} \times \mathbb{S}_{+}^{2}}\left[f_{i}\left(t, x, v^{\prime}\right) f_{j}\left(t, x, w^{\prime}\right)-f_{i}(t, x, v) f_{j}(t, x, w)\right] \\
& \times \Theta\left(\langle\epsilon, v-w\rangle-\Gamma_{i j}\right)\langle\epsilon, v-w\rangle d \epsilon d w,
\end{aligned}
$$


and

$$
\begin{array}{r}
\left.J_{i}^{R}=\beta_{i j} \sigma_{i j}^{2} \iint_{\mathbb{R}^{3} \times \mathbb{S}_{+}^{2}}\left[\left(\frac{\mu_{i j}}{\mu_{k l}}\right)^{3 / 2} f_{k}\left(t, x, v_{i j}^{\odot}\right) f_{l}\left(t, x, w_{i j}^{\odot}\right)-f_{i}(t, x, v), w\right) f_{j}(t, x, w)\right] \\
\times \Theta\left(\langle\epsilon, v-w\rangle-\Gamma_{i j}\right)\langle\epsilon, v-w\rangle d \epsilon d w,
\end{array}
$$

where $f_{i 0}, i=1, \ldots, 4$ are suitable nonnegative initial conditions that will be defined later and $\Omega \subseteq \mathbb{R}^{3}$ denotes the spatial domain of the gas mixture. We consider two choices for the set $\Omega$ :, $\Omega=\mathbb{R}^{3}$, or $\Omega$ being a 3 -dimensional torus $[0, L]^{3}, L>0$. The latter choice corresponds to case of the periodic boundary conditions on $[0, L]^{3}$. Also, $\Gamma_{i j}=\sqrt{2 \gamma_{i} / \mu_{i j}}$ and $\Theta$ is the Heaviside step function. As before, the pairs of postreactive velocities are $\left(v_{i j}^{\odot}, w_{i j}^{\odot}\right)=\left(v^{\ddagger}, w^{\ddagger}\right)$ for $i, j=1,2$, and $\left(v_{i j}^{\odot}, w_{i j}^{\odot}\right)=\left(v^{\dagger}, w^{\dagger}\right)$ for $i, j=3,4$. The pairs of indices $(i, j)$ and $(k, l)$ are from the set of quadruples $(i . j, k, l)$ : $\{(1,2,3,4),(2,1,4,3),(3,4,1,2),(4,3,2,1)\}$.

Proposition 1. Assume that $\beta_{i j}=\beta_{j i}$ for $(i, j) \in\{(1,2),(2,1),(3,4),(4,3)\}$. For $\phi_{i}$ measurable on $\Omega \times \mathbb{R}^{3}$ and $f_{i} \in C_{0}\left(\Omega \times \mathbb{R}^{3}\right), i=1, \ldots, 4$, we have:

$$
\begin{gathered}
\sum_{i=1}^{4} \int_{\mathbb{R}^{3}} \phi_{i} J_{i}^{E} d v=\sum_{i=1}^{4} \sum_{s=1}^{4} \sigma_{i s}^{2} \iint_{\mathbb{R}^{3} \times \mathbb{R}^{3} \times \mathbb{S}_{+}^{2}}\left[\phi_{i}(x, v)+\phi_{s}(x, w)-\phi_{i}\left(x, v^{\prime}\right)-\phi_{s}\left(x, w^{\prime}\right)\right] \times \\
{\left[f_{i}\left(v^{\prime}\right) f_{s}\left(w^{\prime}\right)-f_{i}(v) f_{s}(w)\right]\langle\epsilon, v-w\rangle \Xi_{i s} d \epsilon d w d v,} \\
\sum_{i=1}^{4} \int_{\mathbb{R}^{3}} \phi_{i} J_{i}^{R} d v=\iiint_{\mathbb{R}^{3} \times \mathbb{R}^{3} \times \mathbb{S}_{+}^{2}}\left[\beta_{12} \sigma_{12}^{2} \phi_{1}(x, v)+\beta_{21} \sigma_{21}^{2} \phi_{2}(x, w)-\beta_{34} \sigma_{34}^{2} \phi_{3}\left(x, v^{\ddagger}\right)-\beta_{43} \sigma_{43}^{2} \phi_{4}\left(x, w^{\ddagger}\right)\right] \times \\
{\left[\left(\frac{\mu_{12}}{\mu_{34}}\right)^{3 / 2} f_{3}\left(x, v^{\ddagger}\right) f_{4}\left(x, w^{\ddagger}\right)-f_{1}(x, v) f_{2}(x, w)\right] \Theta\left(\langle\epsilon, v-w\rangle-\Gamma_{12}\right)\langle\epsilon, v-w\rangle d \epsilon d w d v,}
\end{gathered}
$$

where $\Xi_{\text {is }}$, appearing in (23), is given by

$$
\Xi_{i s}= \begin{cases}\frac{1}{2} \Theta\left(\langle\epsilon, v-w\rangle-\Gamma_{i s}\right)+\frac{1}{2}\left(1-\beta_{i s}\right) \Theta\left(\Gamma_{i s}-\langle\epsilon, v-w\rangle\right), & \text { if }(i, s) \in I, \\ \frac{1}{4} \Theta(\langle\epsilon, v-w\rangle), & \text { if } i=s \\ \frac{1}{2} \Theta(\langle\epsilon, v-w\rangle), & \text { otherwise, }\end{cases}
$$

with $I=\{(1,2),(2,1),(3,4),(4,3)\}$.

The post-collisional velocities, $v^{\prime}$ and $w^{\prime}$, are given in (1), while the post-reactive velocities, $v^{\ddagger}$ and $w^{\ddagger}$, are given in (3)-(4).

Due to space limitations, the proof of Proposition 1 will be provided in the forthcoming work. 


\section{Conservation laws}

Under the additional assumption $\beta_{12} \sigma_{12}^{2}=\beta_{34} \sigma_{34}^{2}$, Proposition 1 implies that for any $a, c \in \mathbb{R}$ and $b \in \mathbb{R}^{3}$,

$$
\phi_{i}(x, v)=a m_{i}+m_{i}\langle b, v\rangle+c\left(\frac{m_{i} v^{2}}{2}+E_{i}\right), i=1, \ldots, 4, \Longrightarrow\left\{\begin{array}{l}
\sum_{i=1}^{4} \int_{\mathbb{R}^{3}} \phi_{i} J_{i}^{E} d v=0, \\
\sum_{i=1}^{4} \int_{\mathbb{R}^{3}} \phi_{i} J_{i}^{R} d v=0 .
\end{array}\right.
$$

Property (26) implies that if $f_{i}$ is a nonnegative smooth solution of $(20)$ on $[0, T], T>0$, then, at least formally, we have the following conservation laws for $t \in[0, T]$, relative to mass, momentum and total energy:

$$
\begin{gathered}
\sum_{i=1}^{4} \iint_{\Omega \times \mathbb{R}^{3}} m_{i} f_{i}(t, x, v) d v d x=\sum_{i=1}^{4} \iint_{\Omega \times \mathbb{R}^{3}} m_{i} f_{i 0}(x, v) d v d x \\
\sum_{i=1}^{4} \iint_{\Omega \times \mathbb{R}^{3}} m_{i} v f_{i}(t, x, v) d v d x=\sum_{i=1}^{4} \iint_{\Omega \times \mathbb{R}^{3}} m_{i} v f_{i 0}(x, v) d v d x \\
\sum_{i=1}^{4} \iint_{\Omega \times \mathbb{R}^{3}}\left(\frac{m_{i} v^{2}}{2}+E_{i}\right) f_{i}(t, x, v) d v d x=\sum_{i=1}^{4} \iint_{\Omega \times \mathbb{R}^{3}}\left(\frac{m_{i} v^{2}}{2}+E_{i}\right) f_{i 0}(x, v) d v d x
\end{gathered}
$$

where $f_{i 0}(x, v), i=1, \ldots, 4$, are nonnegative initial conditions of the dilute SRS kinetic system (20). For $f_{i}$, a smooth solution with compact support, the above conservation laws follow easily from multiplying $i$-th equation of the dilute SRS system by $\phi_{i}$, integrating with respect to $(t, x, v) \in[0, T] \times \Omega \times \mathbb{R}^{3}$, and then applying (26).

\section{Entropy identity, $H$-function, and equilibrium solutions}

Proposition 1 also implies existence of a Liapunov functional (an $H$-function) for (20)-(22) consistent with system's physical equilibrium. Assume that for $i, j=1, \ldots, 4$, the conditions $\beta_{i j}=\beta_{j i}$ and $\beta_{12} \sigma_{12}^{2}=\beta_{34} \sigma_{34}^{2}$ are satisfied. For $f_{i}$, a smooth nonnegative solution, we multiply $(20)$ by $1+\log \left(f_{i} / \mu_{i j}\right)$ with $i=1, \ldots 4$ and $(i, j) \in\{(1,2),(2,1),(3,4),(4,3)\}$, integrate over $\Omega \times \mathbb{R}^{3}$, and use (23)-(24) (with $\phi_{i}=\log \left(f_{i} / \mu_{i j}\right)$ ) to obtain the following entropy identity:

$$
\begin{aligned}
& \frac{d}{d t} \sum_{i=1}^{4} \iint_{\Omega \times \mathbb{R}^{3}} f_{i} \log \left(f_{i} / \mu_{i j}\right) d v d x+\sum_{i, s=1}^{4} \sigma_{i s}^{2} \int \ldots \int_{\Omega \times \mathbb{R}^{3} \times \mathbb{R}^{3} \times \mathbb{S}_{+}^{2}}\left[f_{i}\left(v^{\prime}\right) f_{s}\left(w^{\prime}\right)-f_{i}(v) f_{s}(w)\right] \\
& \times \log \left(\frac{f_{i}\left(v^{\prime}\right) f_{s}\left(w^{\prime}\right)}{f_{i}(v) f_{s}(w)}\right)\langle\epsilon, v-w\rangle \Xi_{i s} d \epsilon d w d v d x
\end{aligned}
$$




$$
\begin{aligned}
& +\beta_{12} \sigma_{12}^{2} \int_{\Omega \times \mathbb{R}^{3} \times \mathbb{R}^{3} \times \mathbb{S}_{+}^{2}}\left\{\left[\left(\frac{\mu_{12}}{\mu_{34}}\right)^{3 / 2} f_{3}\left(v^{\ddagger}\right) f_{4}\left(w^{\ddagger}\right)-f_{1}(v) f_{2}(w)\right] \times\right. \\
& \left.\log \left[\left(\frac{\mu_{12}}{\mu_{34}}\right)^{3 / 2} \frac{f_{3}\left(v^{\ddagger}\right) f_{4}\left(w^{\ddagger}\right)}{f_{1}(v) f_{2}(w)}\right] \Theta\left(\langle\epsilon, v-w\rangle-\Gamma_{12}\right)\langle\epsilon, v-w\rangle\right\} d \epsilon d w d v d x=0 .
\end{aligned}
$$

We observe that the second and the third terms in the left hand side of (30) are nonnegative and thus, the convex function $H(t)$, defined by

$$
H(t)=\sum_{i=1}^{4} \iint_{\Omega \times \mathbb{R}^{3}} f_{i}(t, x, v) \log \left[f_{i}(t, x, v) / \mu_{i j}\right] d v d x,
$$

is non-increasing in $t \geq 0$. Hence $H(t)$ is an $H$-function (Liapunov functional) of the system (20)-(22).

For the standard macroscopic quantities (moments of $f_{i}$ ), namely the number densities $n_{i}(t, x)$, the macroscopic velocity $u(t, x)$, and the macroscopic temperature $\mathcal{T}(t, x)$, we have the following characterization of equilibrium solutions for the system (20)-(22):

Proposition 2. Assume that for $i, j=1, \ldots, 4$, the coefficients $0<\beta_{i j} \leq 1$ satisfy the conditions $\beta_{i j}=\beta_{j i}$ and $\beta_{12} \sigma_{12}^{2}=\beta_{34} \sigma_{34}^{2}$. Let $n_{i}(t, x) \geq 0, u(t, x)$, and $\mathcal{T}(t, x) \geq 0$ be given measurable functions. Then for all $0 \leq f_{i} \in L^{1}\left(\Omega \times \mathbb{R}^{3}\right)$, the following statements are equivalent:

1. $f_{i}=n_{i}\left(\frac{m_{i}}{2 \pi k \mathcal{T}}\right)^{3 / 2} \exp \left(-\frac{m_{i}(v-u)^{2}}{2 k \mathcal{T}}\right), i=1, \ldots, 4$,

and $n_{1} n_{2}=\left(\frac{\mu_{12}}{\mu_{34}}\right)^{3 / 2} n_{3} n_{4} \exp \left(\frac{E_{a b s}}{k \mathcal{T}}\right)$;

2. $J_{i}^{E}\left(\left\{f_{i}\right\}\right)=0$ and $J_{i}^{R}\left(\left\{f_{i}\right\}\right)=0, i=1, \ldots, 4$;

3. $\sum_{i=1}^{4} \int_{\mathbb{R}^{3}}\left[J_{i}^{E}\left(\left\{f_{i}\right\}\right)+J_{i}^{R}\left(\left\{f_{i}\right\}\right)\right] \log \left(f_{i} / \mu_{i j}\right) d v=0$.

The proof of Proposition 2 follows a similar line of arguments as the proof of Proposition 3.2 in [5].

\section{Existence result}

Definition 1. A nonnegative $f_{i} \in L_{\text {loc }}^{1}\left((0, T) \times \Omega \times \mathbb{R}^{3}\right), i=1,2,3,4$, is a mild solution of the system (20)-(22) if for each $0<T<\infty$, the gain and loss terms of $J_{i}^{E}$ and $J_{i}^{R}$ are in $L^{1}(0, T)$, a.e. (almost everywhere) in $(x, v) \in \Omega \times \mathbb{R}^{3}$ and

$$
f_{i}^{\#}(t, x, v)-f_{i}^{\#}(s, x, v)=\int_{s}^{t}\left[J_{i}^{E}\left(\left\{f_{i}\right\}\right)^{\#}(\tau, x, v)+J_{i}^{R}\left(\left\{f_{i}\right\}\right)^{\#}(\tau, x, v)\right] d \tau, \quad 0<s<t \leq T,
$$

where $f_{i}^{\#}(t, x, v)=f(t, x+t v, v)$ and with similar definitions for $J_{i}^{E \#}$ and $J_{i}^{R \#}$. 
The following result generalizes Theorem 4.2 of [5] (see also Theorem 5.2 of [6]).

Theorem 1. Assume that for $i, j=1, \ldots, 4$, the coefficients $0 \leq \beta_{i j} \leq 1$ satisfy the conditions $\beta_{i j}=\beta_{j i}$ and $\beta_{12} \sigma_{12}^{2}=\beta_{34} \sigma_{34}^{2}$. If for each $i=1, \ldots, 4$, the initial conditions $f_{i 0} \geq 0$, satisfy

$$
\sup _{i} \iint_{\Omega \times \mathbb{R}^{3}}\left(1+x^{2}+v^{2}+\log ^{+} f_{i 0}\right) f_{i 0} d v d x=C_{0}<\infty,
$$

with $\log ^{+}(z)=\max \{\log (z), 0\}$, then there exists a nonnegative mild solution $\left\{f_{i}\right\}$ of the system (20)-(22), with $f_{i} \in C\left([0, T] ; L^{1}\left(\Omega \times \mathbb{R}^{3}\right)\right)$ and such that $\left.f_{i}(t)\right|_{t=0}=f_{i 0}$, for $i=$ $1,2,3,4$.

The proof of Theorem 1 is similar to Theorem 4.2 of [5] and will not be provided here.

Remark 1. In the case $\beta_{i j}=0$, for $i, j=1, \ldots, 4$, Theorem 1 provides existence result for chemically inert system (20)-(22).

Acknowledgements. The paper is partially supported by CMat-FCT and by the Project FCT-PTDC/MAT/68615/2006.

\section{References}

[1] N. Xystris and J. S. Dahler, J. Chem. Physics, 68, 387-401 (1978).

[2] J. S. Dahler and L. Qin, J. Chem. Physics, 103, 725-750 (1995).

[3] J. S. Dahler and L. Qin, J. Chem. Physics, 118, 8396-8404 (2003).

[4] H. van Beijren and M. H. Ernst, Physica, 68, 437-456 (1973).

[5] J. Polewczak, J. Stat. Physics, 100, 327-362 (2000).

[6] M. Groppi and J. Polewczak, J. Stat. Physics, 117, 211-241 (2004). 